\title{
INCLUSION RELATIONS BETWEEN POWER METHODS AND MATRIX METHODS OF LIMITATION(1)
}

BY

\author{
ABRAHAM ZIV
}

\begin{abstract}
A matrix method of limitation is a generalization of both ordinary Toeplitz methods and semicontinuous methods. A power method is a generalization of both Abel's method and Borel's exponential method. The main concern of this paper is to find necessary and sufficient conditions for the field of a given power method to be included in the field of a given matrix method. The problem is solved for a wide family of power methods which includes all Abel type methods, the logarithmic method, all Borel type methods and others (also nonregular power methods). Preliminary results, which serve as tools in the solution of the main problem, clarify some aspects of the nature of the field of a power method as an FK space.
\end{abstract}

1. Introduction. First let us define a power method of limitation (see Birkholc [2] and Włodarski [17]). Such a method is determined by a power series with complex coefficients, $P(x)=\sum_{k=0}^{\infty} p_{k} x^{k}$, whose radius of convergence, $\rho$ $>0$, is either finite or infinite and which satisfies $P(x) \neq 0$ for $0 \leqslant \alpha \leqslant x$ $<\rho$. Assuming that $\sum p_{k} s_{k} x^{k}$ converges for all $x \in[\alpha, \rho)$ the P-limit of the sequence $s=\left\{s_{k}\right\}$ is defined by

$$
\lim _{p} s=\lim _{x \rightarrow \rho^{-}} T_{p}(s, x), \quad T_{p}(s, x)=\sum_{k=0}^{\infty} p_{k} s_{k} x^{k} / P(x) \quad(\alpha \leqslant x<\rho) .
$$

$T_{p}(s, x)$ is the P-transform of $s$. The best known power methods are those of Abel (with $P(x)=1 /(1-x)$ ) and of Borel (with $P(x)=e^{x}$ ).

Next we define a matrix method of limitation (here we follow M. Lazic [10] with some insignificant differences). Such a method is determined by a subset $I$ of some topological space, a point of accumulation of $I, x_{0}\left(x_{0} \notin I\right)$, which has a denumerable basis of neighborhoods, and a matrix of complex numbers $W=\left(w_{x k}\right)(x \in I, k=0,1, \ldots)$. Assuming that $\sum_{k=0}^{\infty} w_{x k} s_{k}$ converges for

Received by the editors September 5, 1974 and, in revised form, March 16, 1976.

AMS (MOS) subject classifications (1970). Primary 40D25, 40G10, 40H05; Secondary 40C05, 40C15, 40D15, 40D20, 41A10, 41A30, 30A82.

(')The contents of this paper are a part of the author's Ph.D. thesis written under the direction of Professor A. Jakimovski at the Department of Mathematics, Technion, Israel Institute of Technology. The author wishes to thank Professor Jakimovski for his attention and helpful advice. 
all $x \in I$ we define the $W$-limit of $s$ by

$$
\lim _{w} s=\lim _{x \rightarrow x_{0}} T_{w}(s, x), \quad T_{w}(s, x)=\sum_{k=0}^{\infty} w_{x k} s_{k} \quad(x \in I) .
$$

$T_{w}(s, x)$ is the $W$-transform of $s$.

Obviously every power method is also a matrix method.

Let us denote by $c_{p}$ the field of the method $P$, i.e., the set of all sequences which are $P$-convergent to a finite limit, by $c_{p}^{(0)}$ the set of all sequences which are $P$-convergent to zero, and by $m_{p}$ the set of all sequences whose $P$-transforms exist and are bounded. Let us also denote by $c_{w}, c_{w}^{(0)}, m_{w}$ the sets of sequences which are analogously related to the matrix method $W$.

The main objective of this article is to investigate inclusion relations of the type $c_{p} \subseteq c_{w} \cap m_{w}$. It should be observed that in many interesting cases $c_{w} \subseteq m_{w}$. This is so, for example, if $W$ is an ordinary Toeplitz method (i.e., if $\left.I=\{1,2, \ldots\}, x_{0}=+\infty\right)$ or a power method, or any continuous method (see Włodarski [17]). In these cases the said relation is actually equivalent to $c_{p} \subseteq c_{w}$.

In the proofs we rely on the FK nature of $c_{p}$. This nature was realized and used by Zeller [21], [22], Włodarski [17], Ryll-Nardzewski [12], Birkholc [2] and others. The theory of FK spaces as presented in Zeller's [18] and [19] (see also Wilansky [16]) is assumed to be known to the reader.

Representations for linear continuous functionals on $c_{p}$ are of importance and were found by Zeller [21], Włodarski [17] and Birkholc [2]. We present a slightly different representation which appears to be useful. It turns out that the representation of a given continuous linear functional is never unique. This means that the zero functional has nontrivial representations. These representations are characterized in Theorem 2.2.5 which plays an important role in the derivation of the main results.

Let us denote $e=(1,1,1, \ldots), e^{(l)}=(0, \ldots, 0,1,0, \ldots)$ where the single 1 is at the $l$ th place. Many of our results are easier to formulate and prove in cases where the set $U=\{e\} \cup\left\{e^{(l)} \mid l=0,1, \ldots\right\}$ is fundamental in $c_{p}$. The characterization of such cases is therefore of interest, and few theorems are devoted to it.

The problem is actually very close (almost equivalent, in fact) to the problem of perfection of power methods, which was investigated in the past. Thus, Zeller [21] found that Abel's method is perfect. Ryll-Nardzewski [12] proved that Borel's method is perfect. Włodarski and Ryll-Nardzewski [17] proved that $P$ is perfect if $\rho<\infty$ and $p_{k}>0(k=0,1, \ldots)$, and finally, Birkholc investigated the case $\rho=\infty, p_{k}>0(k=0,1, \ldots)$. He found in [2] the connection between the problem of perfection and the so-called $(M)$ 
property of $P(x)$ (see $\$ 2.3 .3$ below) and used it to solve the problem completely in [3] (for the case $\rho=\infty, p_{k}>0$ ).

We slightly generalize those results and formulate them suitable for our purpose. The problem is satisfactorily solved for the case $\rho<\infty$ with $P$ restricted only by the condition $\sum_{k p_{k} \neq 0} 1 / k=\infty$. The case $\rho=\infty$ is more difficult and is satisfactorily solved only for regular power methods with $p_{k} \neq 0$ for almost all $k$.

The main results, which deal with characterization of the relation $c_{p}$ $\subseteq c_{w} \cap m_{w}$, are formulated in two stages. First the matrix methods $W$ that satisfy $c_{p}^{(0)} \subseteq m_{w}$ are characterized. It turns out that they coincide with those which satisfy $c_{p} \subseteq m_{w}$. Then conditions are formulated which characterize the methods $W$ that satisfy in addition $c_{p} \subseteq c_{w}$. These additional conditions are simple in form if $U$ (see above) is fundamental in $c_{p}$. However it is shown that in some interesting cases this is not so. Hence we apply a slightly more complicated, but also more general, approach which enables us to solve the problem in greater generality.

The above-mentioned main results solve the inclusion problem completely with only one restrictive condition which the power method $P$ should satisfy: $\Sigma_{k p_{k} \neq 0} 1 / k=\infty$. The complementary case, $\sum_{k p_{k} \neq 0} 1 / k<\infty$, remains open.

It should be noted that in many instances our proofs could be greatly simplified if the discussion is restricted to regular power methods with $\rho<\infty$. The case $\rho=\infty$ is essentially more difficult (even if $p_{k}>0, k=0,1, \ldots$ ) and nonregularity introduces more difficulties which force the proofs to be longer and more complex.

\section{Definitions and statement of results.}

\subsection{Some definitions.}

2.1.1. Matrix methods of limitation. Let $W=\left(w_{x k}\right)(x \in I, k=0,1, \ldots)$ be an infinite matrix of complex numbers with $I$ a subset of some topological space. Let $x_{0} \notin I$ be a point of accumulation of $I$ which has a denumerable basis of neighborhoods. A sequence $s=\left\{s_{k}\right\}$ of complex numbers is said to be $W$-convergent to $\sigma$ if the series

$$
T_{w}(s, x)=\sum_{k=0}^{\infty} w_{x k} s_{k}
$$

converges for all $x \in I$ and $\lim _{x \rightarrow x_{0}} T_{w}(s, x)=\sigma(x \in I)$. In this case we write $\lim _{w} s=\sigma$. The function $T_{w}(s, x)$ is called the $W$-transform of $s$. We denote by $c_{w}$ the field of the method, i.e., the set of all sequences which are $W$ convergent to a finite limit. By $c_{w}^{(0)}$ we denote the set of all sequences which are $W$-convergent to zero and by $m_{w}$ the set of all sequences whose $W$ transforms exist and are bounded in $I$.

This definition includes as particular cases both the Toeplitz methods, for 
which $I$ is composed of a single sequence that converges to $x_{0}$, and the semicontinuous methods for which $I$ is composed of a finite or an infinite segment of real numbers, one of whose ends is $x_{0}$.

2.1.2. Power methods of limitation. Let $P(x)=\sum_{k=0}^{\infty} p_{k} x^{k}$ be a complex series with radius of convergence $0<\rho \leqslant \infty$ and assume that $P(x) \neq 0$ for $0 \leqslant \alpha \leqslant x<\rho$. A sequence $s=\left\{s_{k}\right\}$ of complex numbers is said to be $P$ convergent to $\sigma$ if $\sum p_{k} s_{k} x^{k}$ is convergent for all $x \in[\alpha, \rho)$ and

$$
\lim _{x \rightarrow \rho^{-}} T_{p}(s, x)=\sigma \quad(x \text { real })
$$

where $T_{p}(s, x)=\sum_{k=0}^{\infty} p_{k} s_{k} x^{k} / P(x)(\alpha \leqslant x<\rho)$ is the $P$-transform of $s$. We write $\sigma=\lim _{p} s$. This is obviously a particular case of a matrix method. The sets $c_{w}^{(0)}, c_{w}, m_{w}$ will be denoted in this case by $c_{p}^{(0)}, c_{p}, m_{p}$. In most of the results of this paper $P$ is required to satisfy the additional condition

$$
\sum_{k p_{k} \neq 0} 1 / k=\infty
$$

This, however, is not considered a part of the edfinition of a power method. The following are examples of some well-known power methods:

$A-$ Abel's method. $P(x)=1 /(1-x), \alpha=0, \rho=1$.

$B$-Borel's exponential method. $P(x)=e^{x}, \alpha=0, \rho=\infty$.

$A_{\lambda}$-Abel type methods. $P(x)=(1-x)^{-1-\lambda}, \lambda>-1, \alpha=0, \rho=1$ (see Jakimovski [1] and Borwein [4]).

L or $A_{-1}$-logarithmic method.

$$
P(x)=\log 1 /(1-x), \quad \alpha>0, \rho=1
$$

(see Borwein [5]).

$(B, a, b)$-Borel type methods.

$$
\begin{array}{r}
P(x)=\sum_{k=N}^{\infty} x^{k} / \Gamma(a k+b) \sim a^{-1} x^{(1-b) / a} \exp \left(x^{1 / a}\right) \quad(x \rightarrow+\infty), \\
a>0,-\infty<b<\infty, a N+b>0, \alpha>0, \rho=\infty
\end{array}
$$

(see Borwein [6]).

2.1.3. Functions of bounded variation. Every function whose variation is bounded in some finite or infinite interval is assumed throughout to be continuous to the right at all points of this interval, with the possible exception of its ends. This convention does not include functions which arise as the outcome of a limiting process. Such functions might be of bounded variation but not continuous to the right.

2.2. $c_{p}$ as an FK space and the representation of continuous linear functionals.

2.2.1. TheOREM. Let $\alpha<\lambda_{0}<\lambda_{1}<\cdots<\lambda_{j} \rightarrow \rho$ and $s \in c_{p}$. The set of seminorms 


$$
\begin{aligned}
Q_{2 i}(s)=\left|s_{i}\right| ; & Q_{2 i+1}(s)=\sum_{k=0}^{\infty}\left|p_{k} s_{k}\right| \lambda_{i}^{k} \quad(i=0,1, \ldots) \\
Q_{\infty}(s)=\sup _{\alpha \leqslant x<\rho}\left|T_{p}(s, x)\right| &
\end{aligned}
$$

defines in $c_{p}$ an FK topology. $c_{p}^{(0)}$ forms a closed, linear subspace of this FK space.

2.2.2. REMARK. It would have simplified matters if a BK topology could be assigned to $c_{p}$. Unfortunately no power method exists for which this is possible.

2.2.3. THEOREM. Every continuous linear functional $f$ in $c_{p}$ has a representation of the form

$$
f(s)=\sum_{k=0}^{\infty} \beta_{k} s_{k}+\int_{\alpha}^{\rho^{-}} T_{p}(s, t) d g(t)+\beta \lim _{p} s \quad\left(s \in c_{p}\right)
$$

where

$$
\begin{aligned}
\left|\beta_{k}\right| \leqslant R\left|p_{k}\right| r^{k}, \quad R<\infty, & r<\rho \\
& (k=m, m+1, \ldots, m \in\{0,1, \ldots\})
\end{aligned}
$$

and $g(t)$ is of bounded variation in $[\alpha, \rho)$. Conversely: Every such a functional is well defined for all $s \in c_{p}$ and is linear and continuous.

A weaker version of the following theorem is proved, using different means in [23]:

2.2.4. THEOREM. Let (2.1.1) be satisfied. If $\sum \beta_{k} s_{k}$ converges for all $s \in c_{p}^{(0)}$ then (2.2.2) is satisfied.

2.2.5. THEOREM. Let (2.1.1) be satisfied and let $f(s)$ be defined by (2.2.1), where $g$ is of bounded variation in $[\alpha, \rho)$. (a) $f$ is well defined and vanishes for all $s \in c_{p}^{(0)}$ if and only if

(i) $g=$ const in $[r, \rho)$ for some $r<\rho$.

(ii) $\beta_{k}=-p_{k} \int_{\alpha}^{\rho^{-}}\left(t^{k} / P(t)\right) d g(t)(k=0,1, \ldots)$.

(b) $f$ is well defined and vanishes for all $s \in c_{p}$ if and only if (i), (ii) and

(iii) $\beta=0$.

2.2.6. REMARK. Observe that the sufficiency part of Theorem 2.2 .5 is valid even if (2.1.1) is not satisfied.

2.3. The sequences $e^{(l)}$ in $c_{p}$.

2.3.1. The sequences $e^{(l)}, e$ and the sets $U, U^{\prime}$. We denote $e=(1,1,1, \ldots)$ and $e^{(l)}=(0, \ldots, 0,1,0, \ldots)$ where the single 1 is at the $l$ th place. Also $U=\{e\} \cup\left\{e^{(l)} \mid l=0,1, \ldots\right\}$ and $U^{\prime}=\left\{e^{(l)} \mid l=0,1, \ldots\right\}$.

2.3.2. THEOREM. Let (2.1.1) be satisfied and let $\rho<\infty$ and $e^{(l)} \in c_{p}(l$ 
$=0,1, \ldots)$. (a) If $e^{(l)} \in c_{p}^{(0)}(l=0,1, \ldots)$ then $U^{\prime}$ is a fundamental set in $c_{p}^{(0)}$ and $U$ is fundamental in $c_{p}$. (b)If $e^{(l)} \notin c_{p}^{(0)}$ for at least one $l$ then $U^{\prime}$ is fundamental in $c_{p}$.

2.3.3. The $\left(M\left\{k_{i}\right\}\right)$ property. Let $0 \leqslant k_{0}<k_{1}<\cdots<k_{i} \rightarrow \infty$ be integers and let $\phi(t)$ be complex valued and continuous in $[\alpha, \rho) . \phi$ is said to have the $\left(M\left\{k_{i}\right\}\right)$ property in $[\alpha, \rho)$ if

$$
\sup _{\alpha \leqslant t<\rho}\left|t^{k_{i}} / \phi(t)\right|<\infty \quad(i=0,1, \ldots)
$$

and the condition

$$
\int_{\alpha}^{\rho^{-}}\left(t^{k_{i}} / \phi(t)\right) d g(t)=O\left(r^{k_{i}}\right), \quad \alpha<r<\rho, \quad \int_{\alpha}^{\rho^{-}}|d g(t)|<\infty
$$

is satisfied only if $g=$ const in $[r, \rho)$. In particular, we shall write $(M)$ instead of $\left(M\left\{k_{i}\right\}\right)$ in the simplest case $k_{i}=i$, or more generally if $k_{i+1}=k_{i}+1$ for all sufficiently large $i$.

2.3.4. THEOREM. Let $\rho=\infty, e^{(l)} \in c_{p}(l=0,1, \ldots)$ and denote by $0<k_{0}$ $<k_{1}<\cdots$ the sequence $\left\{k_{i}\right\}=\left\{k \mid p_{k} \neq 0\right\}$, which is assumed to be infinite. In order that $U$ be fundamental in $c_{p}$ it suffices that $P(t)$ has the $\left(M\left\{k_{i}\right\}\right)$ property in $[\alpha,+\infty)$. If $(2.1 .1)$ holds, this property is also necessary.

2.3.5. Theorem. Let $P$ be a regular power method with $\rho=\infty$ and $p_{k}$ $\neq 0(k=N, N+1, \ldots)$. The condition

$$
\int^{\infty}\left(\ln |P(t)| / t^{3 / 2}\right) d t=\infty
$$

is necessary and sufficient for $U$ to be fundamental in $c_{p}$.

2.3.6. Corollary. $U$ is fundamental in the fields of the methods $A_{\lambda}(\lambda>-1)$ and $L$. It is fundamental in the field of the method $(B, a, b)(a>0,-\infty<b$ $<\infty)$ if and only if $a \leqslant 2$. In particular it is fundamental in the fields of the Abel $\left(A_{\lambda}\right.$ with $\left.\lambda=0\right)$ and Borel $((B, a, b)$ with $a=b=1)$ methods.

2.3.7. ReMARK. Considering the interesting cases in which $U$ is fundamental in $c_{p}$ the question arises whether this set constitutes a Schauder basis in $c_{p}$. Unfortunately this is not so and we prove the following: Let (2.1.1) be satisfied and assume $e^{(l)} \in c_{p}(l=0,1, \ldots)$. Neither $U^{\prime}$ nor $U$ is a Schauder basis in $c_{p}$.

To complete this set of theorems, we cite the following result due to Birkholc, which is used later to deduce Theorem 2.3.5 from Theorem 2.3.4 and might be helpful in other cases also.

2.3.8. TheOREM (BIRKHOLC [3]). Let $\phi(t)$ be real valued, positive and contin- 
uous in $[0, \infty)$ and satisfy (2.3.1) with $\rho=\infty$. If $\phi$ has the $(M)$ property in $[0, \infty)$ it is necessary that

$$
\int_{-\infty}^{\infty}\left[\ln \phi\left(t^{2}\right) /\left(1+t^{2}\right)\right] d t=\infty
$$

If $\phi$ is an entire function of the form

$$
\phi(t)=\sum_{k=0}^{\infty} \phi_{k} t^{k}, \quad \phi_{0}>0, \phi_{k} \geqslant 0(k=1,2, \ldots)
$$

then (2.3.4) is also sufficient.

It is of some interest to note the following:

2.3.9. REMARK. Let $\rho=\infty$ and let $\phi_{1}(t), \phi_{2}(t)$ be complex valued and continuous. If $\phi_{1}(t)$ has the $\left(M\left\{k_{i}\right\}\right)$ property and $[\varepsilon \alpha,+\infty)$ and $\phi_{1}(\varepsilon t) / \phi_{2}(t)$ is bounded in $[\alpha,+\infty)$ for some $\varepsilon>0$ then $\phi_{2}$ has the $\left(M\left\{k_{i}\right\}\right)$ property in $[\alpha,+\infty)$.

2.4. Inclusion between power methods and matrix methods.

2.4.1. ThEOREM. Let (2.1.1) be satisfied. If $c_{p}^{(0)} \subseteq m_{w}$ then the matrix $W$ may be decomposed in the form $W=C+D$, where the matrices $C=\left(c_{x k}\right), D$ $=\left(d_{x k}\right)(x \in I, k=0,1, \ldots)$ satisfy:

(i) $c_{x k}=p_{k} \int_{\alpha}^{r_{x}}\left(t^{k} / P(t)\right) d g_{x}(t), \int_{\alpha}^{r_{x}}\left|d g_{x}(t)\right| \leqslant R<\infty, \alpha<r_{x}<\rho(x \in I$, $k=0,1, \ldots$ ).

(ii) $\left|d_{x k}\right| \leqslant R\left|p_{k}\right| r^{k}, \alpha<r<\rho(x \in I, k=m, m+1, \ldots, m \in\{0,1$, $\ldots\}),\left|d_{x k}\right| \leqslant R(x \in I, k=0,1, \ldots)$,

and $r_{x}, g_{x}$ are independent of $k$, and $R, r, m$ are independent of both $k$ and $x$.

2.4.2. REMARK. Observe that (i), (ii) of Theorem 2.4.1 suffice for the relation $m_{p} \subseteq m_{w}$. This is true even if (2.1.1) is not satisfied.

2.4.3. Corollary. Let (2.1.1) be satisfied. If $c_{p}^{(0)} \subseteq m_{w}$ then $c_{p} \subseteq m_{p} \subseteq m_{w}$.

Birkholc proved [2, Theorem 10] that no perfect power method $P$ exists which satisfies: $p_{k}>0(k=0,1, \ldots), \lim _{x \rightarrow p^{-}} P(x)=\infty$ and $c_{p} \subseteq c_{w}$ for some raw-finite and regular Toeplitz method $W$. The following is a generalization:

2.4.4. THEOREM. Let (2.1.1) be satisfied. No raw-finite and regular matrix method $W$ exists such that $c_{p}^{(0)} \subseteq m_{w}$.

Zeller prove [22, Satz 1] that no series-to-sequence, raw-finite and regular Toeplitz method exists which sums all the Abel summable series. This is generalized by the following corollary of Theorem 2.4.4.

2.4.5. COROLLARY. Let (2.1.1) be satisfied. No raw-finite and regular series-to- 
sequence Toeplitz method exists which sums all the P-summable series.

2.4.6. TheOREM. Let $U$ be fundamental in $c_{p}$ and assume that $c_{p} \subseteq m_{w}$. In order that $c_{p} \subseteq c_{w}$ it is necessary and sufficient that

(iii) The limit $\lim _{x \rightarrow x_{0}} w_{x k}(x \in I)$ exists and is finite for all $k \in\{0,1, \ldots\}$.

(iv) The limit $\lim _{x \rightarrow x_{0}} \sum_{k=0}^{\infty} w_{x k}(x \in I)$ exists and is finite.

If $U^{\prime}$ is fundamental in $c_{p}$ (see Theorem 2.3.2) then (iii) is already sufficient.

2.4.7. REMARK. In order to show how essential is the fundamentality of $U$ for the validity of the previous theorem we prove the following:

Let (2.1.1) be satisfied and let $U$ be included in $c_{p}$ but not fundamental there. There exists a matrix method $W$, which satisfies conditions (i), ..., (iv) of Theorems 2.4.1 and 2.4.6 but $c_{p} \& c_{w}$.

2.4.8. Corollary. In order that $c_{a} \subseteq m_{w} \cap c_{w}$ ( $c_{a}$ is the field of Abel's method) it is necessary and sufficient that $W=C+D$ where

(i) $c_{x k}=\int_{0}^{r_{x}}(1-t) t^{k} d g_{x}(t), \quad \int_{0}^{r_{x}}\left|d g_{x}(t)\right|<R, 0<r_{x}<1 \quad(x \in I, k=$ $0,1, \ldots)$,

(ii) $)_{a}\left|d_{x k}\right| \leqslant R r^{k}, r<1(x \in I, k=0,1, \ldots)$,

and conditions (iii) and (iv) of Theorem 2.4.6 hold.

2.4.9. COROLlaRY. In order that $c_{b} \subseteq m_{w} \cap c_{w}\left(c_{b}\right.$ is the field of Borel's method) it is necessary and sufficient that $W=C+D$ where

(i) $c_{x k}=(1 / k !) \int_{0}^{r_{x}} e^{-t} t^{k} d g_{x}(t), \int_{0}^{r_{x}}\left|d g_{x}(t)\right|<R, 0<r_{x}<+\infty(x \in I, k$ $=0,1, \ldots)$,

(ii) ${ }_{b}\left|d_{x k}\right|<R r^{k} / k !, r<\infty(x \in I, k=0,1, \ldots)$.

and the conditions (iii) and (iv) of Theorem 2.4 .6 hold.

2.4.10. Corollary. Let $U$ be fundamental in $c_{p}$ and let $C=\left(c_{x k}\right)$ satisfy (i) of Theorem 2.4.1. In order that $c_{p} \subseteq c_{c}$ it is necessary and sufficient that

(iii) The limit $\lim _{x \rightarrow x_{0}} c_{x k}(x \in I)$ exists and is finite for all $k \in\{0,1, \ldots\}$.

(iv) The limit $\lim _{x \rightarrow x_{0}} \sum_{k=0}^{\infty} c_{x k}(x \in I)$ exists and is finite.

If $U^{\prime}$ is fundamental in $c_{p}$ then (iii) ${ }_{c}$ is already sufficient.

Theorems 2.4.1 and 2.4.6 give conditions for the inclusion $c_{p} \subseteq m_{w} \cap c_{w}$ in case $U$ is fundamental in $c_{p}$. Remark 2.4 .7 convinces us that the same conditions do not suffice if $U$ is not fundamental in $c_{p}$. However according to Theorem 2.3.5 and Corollary 2.3.6 there exist interesting cases (such as $(B, a, b)$ with $a>2)$ where $U$ is not fundamental in $c_{p}$. In order to include these cases we present below another, more general, approach to the inclusion problem.

2.4.11. Accumulation system. Let $g=g(t)(\alpha<t<\rho<\infty)$ be some complex valued function and $d=\left\{d_{k}\right\}$ be some complex sequence. The couple $(g, d)$ forms a system. Let $\left\{\left(g_{x}, d_{x}\right) \mid x \in I\right\}$, where $g_{x}=g_{x}(t)$ and $d_{x}=\left\{d_{x k}\right\}$, be a set of systems. $(g, d)$ is called an accumulation system of the 
said set, at $x_{0}$, if a sequence $\left\{x_{n}\right\}$ exists which satisfies: $x_{n} \in I(n=$ $1,2, \ldots), x_{n} \rightarrow x_{0}, g_{x_{n}}(t) \rightarrow g(t), d_{x_{n}, k} \rightarrow d_{k}$, for all $\alpha \leqslant t \leqslant \rho$ and $k \in$ $\{0,1, \ldots\}$. In this case $g$ is called the accumulation function of $\left\{g_{x} \mid x \in I\right\}$ at $x_{0}$, and $d$ is called the accumulation sequence of $\left\{d_{x} \mid x \in I\right\}$ at $x_{0}$.

2.4.12. ThEOREM. Let $P$ and $W$ satisfy all the conditions mentioned in Theorem 2.4.1 and assume further that $g_{x} \equiv 0$ in $\left[r_{x}, \rho\right]$, for all $x \in I$. In order that $c_{p} \subseteq c_{w}$ it is necessary and sufficient that a function $\tilde{g}=\tilde{g}(t)$ and a sequence $\tilde{d}=\left\{\tilde{d}_{k}\right\}$ exist, which satisfy for every accumulation system $(g, d)$ of $\left\{\left(g_{x}, d_{x}\right) \mid\right.$ $x \in I\}$ at $x_{0}$ the conditions

(i) $g(t)=\tilde{g}(t)$ almost everywhere in $(r, \rho)$,

(ii) $p_{k} \int_{\alpha}^{r_{+}}\left(t^{k} / P(t)\right) d g(t)+d_{k}=\tilde{d}_{k}(k=0,1, \ldots)$.

In this case every accumulation system $(g, d)$ satisfies

$$
\begin{aligned}
\lim _{w} s & =\sum_{k=0}^{\infty} d_{k} s_{k}+\int_{\alpha}^{\rho^{-}} T_{p}(s, t) d g(t)-g(\rho-0) \lim _{p} s \\
& =\sum_{k=0}^{\infty} \tilde{d}_{k} s_{k}+\int_{r^{+}}^{\rho^{-}} T_{p}(s, t) d g(t)-g(\rho-0) \lim _{p} s \quad\left(s \in c_{p}\right), \\
\int_{\alpha}^{\rho^{-}} & |d g(t)|+|g(\rho-0)| \leqslant R \\
& \left|d_{0}\right|,\left|d_{1}\right|, \ldots \leqslant R,\left|d_{k}\right| \leqslant R\left|p_{k}\right| r^{k} \quad(k=m, m+1, \ldots) .
\end{aligned}
$$

2.4.13. RemarK. It is natural to ask whether condition (i) of the last theorem may be replaced by the simpler condition:

$$
\lim _{x \rightarrow x_{0}} g_{x}(t)=\tilde{g}(t) \quad(x \in I) \text { almost everywhere in }[r, \rho) .
$$

Unfortunately this is not so even in the simplest case where $W$ is a Toeplitz method. A counterexample is given in $\$ 5.12$.

2.4.14. Corollary. Let (2.1.1) be satisfied and let $C, D$ be matrices which satisfy conditions (i), (ii) of Theorem 2.4.1. Assume further that $g_{x} \equiv 0$ in $\left[r_{x}, \rho\right]$ for every $x \in I$. (a) $c_{p} \subseteq c_{c}$ if and only if every two accumulation functions of $\left\{g_{x} \mid x \in I\right\}$ at $x_{0}$ are equal to each other at $t=\alpha$ and almost everywhere in $(\alpha, \rho)$. In this case

$$
\lim _{c} s=\int_{\alpha}^{\rho^{-}} T_{p}(s, t) d g(t)-g(\rho-0) \lim _{p} s \quad\left(s \in c_{p}\right)
$$

where $g$ is any accumulation function of $\left\{g_{x} \mid x \in I\right\}$ at $x_{0}$. (b) $c_{p} \subseteq c_{d}$ if and only if all the limits $d_{k}=\lim _{x \rightarrow x_{0}} d_{x k}(x \in I, k=0,1, \ldots)$ exist and are finite. In this case 


$$
\lim _{d} s=\sum_{k=0}^{\infty} d_{k} s_{k} \quad\left(s \in c_{p}\right)
$$

2.4.15. THEOREM. Let (2.1.1) be satisfied. In order that $c_{p} \subseteq m_{w} \cap c_{w}$ it is necessary and sufficient that $W$ is decomposable in the form $W=C+D$ where $C$ and $D$ satisfy conditions (i) and (ii) of Theorem 2.4.1 and $c_{p} \subseteq c_{c} \cap c_{d}$ (see Corollaries 2.4.10 and 2.4.14).

3. Proofs of the results in $\$ 2.2$.

3.1. Proof of Theorem 2.2.1. The proof is of a standard nature and will be omitted.

3.2. Proof of Remark 2.2.2. We generalize an idea of Zeller (see [20, Satz 8.4]). Assuming that $c_{p}$ is a BK space there exist a natural number $n$ and real positive constants $M_{j}(j=\infty, 0,1,2, \ldots)$ such that

$$
Q_{j}(s) \leqslant M_{j}\left(Q_{\infty}(s)+\sum_{k=0}^{2 n} Q_{k}(s)\right) \quad\left(s \in c_{p}, j=\infty, 0,1,2, \ldots\right) .
$$

Define a sequence $s^{\prime} \in c_{p}$ by

$$
s_{k}^{\prime}=\left\{\begin{array}{ll}
k M_{2 k}, & p_{k}=0, \\
0, & p_{k} \neq 0,
\end{array} \quad(k=0,1, \ldots) .\right.
$$

From (3.1), $\sup _{p_{k}=0}\{k\}=\sup _{k}\left\{Q_{2 k}\left(s^{\prime}\right) / M_{2 k}\right\}<\infty$. Hence the equality $p_{k}$ $=0$ cannot hold infinitely many times. Assume, then, that $p_{k} \neq 0$ for $k=m$, $m+1, \ldots$.

Let us use now Lemma 3.7. This is legitimate since its proof is independent of Remark 2.2.2:

We choose $\left\{k_{i}\right\}=\{m, m+1, \ldots\}, \varepsilon>0, \lambda_{n} \leqslant r<\rho, \varepsilon(x)$ such that $\varepsilon(x) / P(x) \rightarrow 0\left(x \rightarrow \rho^{-}\right)$and

$$
h(x)=\left\{\begin{array}{ll}
P(r) x^{m} e^{-\beta x}, & 0 \leqslant x \leqslant r, \\
P(x) x^{m} e^{-\beta x}, & r \leqslant x<\rho,
\end{array} \quad \beta>0 .\right.
$$

The resulting $H(x)$ is used to define uniquely a sequence $s$ (dependent on $\beta$ ) by $s_{0}=s_{1}=\cdots=s_{m-1}=0, P(x) T_{p}(s, x)=\sum p_{k} s_{k} x^{k} \equiv H(x)$.

(3.4) implies, then, that $s \in c_{p}$. It may also be shown (use (3.3) and (3.4)) that $Q_{\infty}(s)$ remains bounded as $\beta \rightarrow+\infty$, that $Q_{2 i}(s)$ does not increase faster than a polynomial in $\beta$, and that $Q_{2 i+1}(s) \sim|P(r)| \lambda_{i}^{m} e^{\beta \lambda_{i}}(i \leqslant n, \beta \rightarrow+\infty)$. This leads to a violation of (3.1) for $j=2 n+1$ and sufficiently large $\beta$.

3.3. LEMMA. Let $f_{j}(s)$ be linear functionals, over a linear subspace $E$ of $c_{p}$, which satisfy the conditions $\left|f_{j}(s)\right| \leqslant M_{j} Q_{j}(s)(s \in E)$.

(a) If $j=2 i(i=0,1, \ldots)$ then 


$$
f_{j}(s)=\beta^{(j)} s_{i}, \quad\left|\beta^{(j)}\right| \leqslant M_{j}(s \in E) .
$$

(b) If $j=2 i+1(i=0,1, \ldots)$ then

$$
f_{j}(s)=\sum_{k=0}^{\infty} \beta_{k}^{(j)} s_{k}, \quad\left|\beta_{k}^{(j)}\right| \leqslant M_{j}\left|p_{k}\right| \lambda_{i}^{k}(s \in E, k=0,1, \ldots) .
$$

(c) $f_{\infty}(s)=\int_{\alpha}^{\rho^{-}} T_{p}(s, t) d g(t)+\beta \lim _{p} s, \int_{\alpha}^{\rho^{-}}|d g(t)|+|\beta|<M_{\infty}$.

Proof. The proof is of a standard nature and will be omitted.

3.4. Proof of Theorem 2.2.3. Let $f$ be a continuous linear functional over $c_{p}$. By Theorem 3.4 of Zeller's [18], $f$ is decomposable in the form

$$
f(s)=f_{\infty}(s)+\sum_{k=0}^{2 n} f_{k}(s), \quad\left|f_{j}(s)\right| \leqslant M Q_{j}(s)\left(s \in c_{p}, j=\infty, 0,1, \ldots, 2 n\right)
$$

and the existence of the representation (2.2.1) follows from Lemma 3.3. The continuity of every such representation is obvious.

The following generalization of the theorem of bounded moments of Mikusinski [11] is due to R. Trautner [14] and plays an important role in our proofs.

3.5. TRAutNeR's Theorem $(\operatorname{SeE}[14])(2)$. Let

$$
\mu_{k}=\int_{0}^{1} \tau^{k} d \chi(\tau), \quad \int_{0}^{1}|d \chi(\tau)|<\infty(k=0,1, \ldots)
$$

be Hausdorff moments (with complex valued $\chi)$. If $\mu_{k_{i}}=O\left(r^{k_{i}}\right)(i \rightarrow \infty, 0<r$ $<1, k_{i} \uparrow \infty, k_{i}$ natural numbers) then either $\chi=$ const in $(r, 1]$ or $\sum_{i} 1 / k_{i}$ $<\infty$.

3.6. Lemma. Let $0 \leqslant k_{0}<k_{1}<\cdots<k_{i} \rightarrow \infty$ be integers such that $\sum_{i=1}^{\infty} 1 / k_{i}=\infty$ and let $\varepsilon>0,0<r<\rho<\infty$. If $h(x)$ is a continuous, complex valued function in $[0, \rho]$ and satisfies

$$
h(x)=\sum_{i=0}^{\infty} c_{i} x^{k_{i}}, \quad \sum_{i=0}^{\infty}\left|c_{i}\right| r^{k_{i}}<\infty(0 \leqslant x \leqslant r),
$$

then a polynomial $H(x)=\sum_{k=0}^{\infty} C_{i} x^{k_{i}}$ exists $\left(C_{i} \neq 0\right.$ only finitely many times $)$ which satisfies

$$
\sum_{i=0}^{\infty}\left|C_{i}-c_{i}\right| r^{k_{i}}<\varepsilon, \quad \sup _{r \leqslant x \leqslant \rho}|H(x)-h(x)|<\varepsilon, H(\rho)=h(\rho) .
$$

(2) The referee attracted the author's attention to a paper by R. P. Boas (Studia Math. 13 (1953), 59-61) which includes similar results. 
Proof. We denote by $E$ the space of all the complex functions $h(x)$ which are continuous in $[0, \rho]$ and satisfy (3.2). This obviously becomes a Banach space by introducing the norm

$$
q(h)=q_{1}(h)+q_{2}(h), \quad q_{1}(h)=\sum_{k=0}^{\infty}\left|c_{i}\right| r^{k_{i}}, q_{2}(h)=\sup _{r<x<\rho}|h(x)| .
$$

The proof will almost be completed if we show that the set $\left\{x^{k_{0}}, x^{k_{1}}, \ldots\right\}$ is fundamental in $E$. This fundamentality is shown by proving that every linear, continuous functional, which vanishes on the said set, vanishes everywhere in $E$. Thus we show first, following the method of proof of Theorem 2.2.3, that every continuous, linear functional over $E$ has a representation of the form

$$
\begin{gathered}
f(h)=\sum_{i=0}^{\infty} \alpha_{i} c_{i}+\int_{r}^{\rho} h(t) d g(t), \\
\left|\alpha_{l}\right| \leqslant R r^{k_{l}} \quad(l=0,1, \ldots), \quad \int_{r}^{\rho}|d g(t)|<\infty .
\end{gathered}
$$

Assuming that $f(h)=0$ for $h(x)=x^{k_{l}}$ we get

$$
\alpha_{l}+\int_{r}^{\rho} t^{k_{l}} d g(t)=0 \Rightarrow\left|\int_{r}^{\rho} t^{k_{l}} d g(t)\right| \leqslant R r^{k_{l}} \quad(l=0,1, \ldots) .
$$

Trautner's theorem implies, then, that $g=$ const in $(r, \rho]$ and therefore, for all $h \in E$,

$$
\begin{aligned}
f(h) & =\sum_{i=0}^{\infty} \alpha_{i} c_{i}+\int_{r}^{r+} h(t) d g(t) \\
& =\sum_{i=0}^{\infty} \alpha_{i} c_{i}+(g(r+0)-g(r)) \sum_{i=0}^{\infty} c_{i} r^{k_{i}} \\
& =\sum_{i=0} \beta_{i} c_{i} .
\end{aligned}
$$

Substituting again $h(x)=x^{k_{l}}$ we get $\beta_{l}=0(l=0,1, \ldots)$ which means that $f(h)=0$ for all $h \in E$, so the fundamentality of proved.

Given $h \in E$ there exists, then, a polynomial $G(x)=\sum_{i=0}^{\infty} D_{i} x^{k_{i}}$ which satisfies $q(G-h)<\varepsilon / 2$. Hence

$$
\sum_{i=0}^{\infty}\left|D_{i}-c_{i}\right| r^{k_{i}}<\varepsilon / 2, \quad \sup _{r \leqslant x \leqslant \rho}|G(x)-h(x)|<\varepsilon / 2 .
$$

Define $H(x)=G(x)+(h(\rho)-G(\rho))(x / \rho)^{k_{0}}$ and we easily see that $H(x)$ satisfies all the necessary conditions.

3.7. LEMMA. Let $0 \leqslant k_{0}<k_{1}<\cdots<k_{i} \rightarrow \infty$ be integers such that $\sum_{i=1}^{\infty} 1 / k_{i}=\infty$ and let $\varepsilon>0,0<r<\rho \leqslant \infty$ be given real numbers and 
$\varepsilon(x)>0$ a real valued function, continuous in $[r, \rho)$. If $h(x)$ is complex valued and continuous in $[0, \rho)$ and satisfies (3.2) there exists a function $H(z)=\sum_{i=0}^{\infty} C_{i} z^{k_{i}}$, analytic in $|z|<\rho$, which satisfies

$$
\begin{array}{r}
\sup _{0<x \leqslant r}|H(x)-h(x)| \leqslant \sum_{i=0}^{\infty}\left|C_{i}-c_{i}\right| r^{k_{i}}<\varepsilon, \\
|H(x)-h(x)|<\varepsilon(x) \quad(r \leqslant x<\rho) .
\end{array}
$$

Proof. The function $H(z)$ will be defined as the limit of a sequence of polynomials $H_{n}(z)=\sum_{i=0}^{\infty} C_{n i} z^{k_{i}}(n=1,2, \ldots)$. We choose first, arbitrarily for the time being, numbers $\varepsilon_{n}>0(n=1,2, \ldots)$ and $r=r_{0}<r_{1}<\ldots$ $<r_{n} \rightarrow \rho$ and put

$$
H_{0}(z)=\sum_{i=0}^{\infty} C_{0 i} z^{k_{i}}=\sum_{i=0}^{\infty} c_{i} z^{k_{i}}=h(z) \quad\left(|z| \leqslant r_{0}\right) .
$$

We proceed then to define $H_{1}(z), H_{2}(z), \ldots$ successively so that the following is satisfied

$$
\begin{gathered}
\sup _{|z| \leqslant r_{n-1}}\left|H_{n}(z)-H_{n-1}(z)\right| \leqslant \sum_{i=0}^{\infty}\left|C_{n i}-C_{n-1, i}\right| r_{n-1}^{k_{i}}<\varepsilon_{n} \\
\quad(n=1,2, \ldots) ; \\
\sup _{r_{n-1} \leqslant x \leqslant r_{n}}\left|H_{n}(x)-h(x)\right|<\varepsilon_{n} \quad(n=1,2, \ldots), \\
H_{n}\left(r_{n}\right)=h\left(r_{n}\right) \quad(n=0,1, \ldots) .
\end{gathered}
$$

The existence of $H_{n}$ follows from the existence of $H_{n-1}$ because we may define

$$
h_{n}(x)= \begin{cases}H_{n-1}(x), & 0 \leqslant x \leqslant r_{n-1}, \\ h(x), & r_{n-1} \leqslant x \leqslant r_{n},\end{cases}
$$

and choose $H_{n}$ as the polynomial approximating $h_{n}(x)$ in $\left[0, r_{n}\right]$, by Lemma 3.6.

We may assume now that $\varepsilon_{n}=E\left(r_{n}\right)-E\left(r_{n+1}\right)$ where $E(x)$ is any real valued, strictly decreasing function which satisfies $E\left(r_{1}\right)<\varepsilon, E(\rho-0)=0$, $E(x)<\varepsilon(x)(r \leqslant x<\rho)$. Now,

$$
H_{j}(z)=H_{1}(z)+\sum_{n=2}^{j}\left(H_{n}(z)-H_{n-1}(z)\right) .
$$

For $|z|<r_{n-1}$ we get from (3.5) that $\left|H_{n}(z)-H_{n-1}(z)\right|<\varepsilon_{n}$, and since $\sum_{n} \varepsilon_{n}=\sum_{n}\left(E\left(r_{n}\right)-E\left(r_{n+1}\right)\right)<\infty$ it follows that $\sum_{n=2}^{\infty}\left(H_{n}(z)-H_{n-1}(z)\right)$ is uniformly convergent in $|z|<M$, provided $M<\rho$. This means that for all $M<\rho$ the sequence $H_{j}(z)$ is uniformly convergent in $|z|<M$ and therefore 
the limit $H(z)=\lim _{j \rightarrow \infty} H_{j}(z)$ exists and is analytic in $|z|<\rho$. Let us see that $H(z)$ satisfies both (3.3) and (3.4):

By Weierstrass' theorem on double series (see Knopp [8, p. 430]) and by (3.5)

$$
\begin{aligned}
\sum_{i=0}^{\infty}\left|C_{i}-c_{i}\right| r^{k_{i}} & =\sum_{i=0}^{\infty}\left|\lim _{n \rightarrow \infty} C_{n i}-C_{0 i}\right| r^{k_{i}} \\
& =\sum_{i=0}^{\infty}\left|\sum_{j=1}^{\infty}\left(C_{j i}-C_{j-i, i}\right)\right| r^{k_{i}} \leqslant \sum_{j=1}^{\infty} \sum_{i=0}^{\infty}\left|C_{j i}-C_{j-1, i}\right| r^{k_{i}} \\
& \leqslant \sum_{j=1}^{\infty} \sum_{i=0}^{\infty}\left|C_{j i}-C_{j-1, i}\right| r_{j-1}^{k_{i}}<\sum_{j=1}^{\infty} \varepsilon_{j} \\
& =\sum_{j=1}^{\infty}\left(E\left(r_{j}\right)-E\left(r_{j+1}\right)\right)=E\left(r_{1}\right)<\varepsilon .
\end{aligned}
$$

This proves (3.3).

Let $r \leqslant x<\rho$. There exists some $n$ for which $r_{n-1} \leqslant x \leqslant r_{n}$. By (3.5) and (3.6),

$$
\begin{aligned}
|H(x)-h(x)| & \leqslant\left|H_{n}(x)-h(x)\right|+\left|H(x)-H_{n}(x)\right| \\
& =\left|H_{n}(x)-h(x)\right|+\left|\sum_{j=n+1}^{\infty}\left(H_{j}(x)-H_{j-1}(x)\right)\right| \leqslant \varepsilon_{n}+\sum_{j=n+1}^{\infty} \varepsilon_{j} \\
& =\sum_{j=n}^{\infty}\left(E\left(r_{j}\right)-E\left(r_{j+1}\right)\right)=E\left(r_{n}\right) \leqslant E(x)<\varepsilon(x) .
\end{aligned}
$$

Hence (3.4) is true also.

3.8. Lemma. Let (2.1.1) be satisfied and let $n \in\{0,1, \ldots\}, \varepsilon>0, \alpha \leqslant r$ $<\rho, S_{0}, S_{1}, \ldots, S_{n-1}$ be given. Assume that $T(x)$ is any function, continuous in $[r, \rho)$, having a finite limit $T(\rho-0)$ and satisfying

$$
T(r)=\sum_{k=0}^{n-1} p_{k} S_{k} r^{k} / P(r)
$$

and let $\delta(x)>0$ be continuous in $[r, \rho)$. There exists a sequence $s \in c_{p}$ which satisfies

$$
\begin{gathered}
s_{j}=S_{j} \quad(j=0,1, \ldots, n-1) ; \quad \sum_{k=n}^{\infty}\left|p_{k} s_{k}\right| r^{k}<\varepsilon, \\
\left|T_{p}(s, x)-T(x)\right|<\delta(x) \quad(r \leqslant x<\rho), \quad \lim _{p} s=T(\rho-0) .
\end{gathered}
$$

Proof. We substitute, in Lemma 3.7, $\varepsilon(x)=\delta(x)|P(x)|,\left\{k_{i}\right\}=\{k \mid k$ $\geqslant n$ and $\left.p_{k} \neq 0\right\}$ 


$$
h(x)=\left\{\begin{array}{l}
0, \quad 0 \leqslant x<r, \\
P(x) T(x)-\sum_{k=0}^{n-1} p_{k} S_{k} x^{k}, \quad r \leqslant x<\rho,
\end{array}\right.
$$

and deduce the existence of a function $H(z)=\sum_{k=n}^{\infty} C_{k} z^{k}$, analytic in $|z|<\rho$, which satisfies: $C_{k}=0$ if $p_{k}=0, \sum_{k=n}^{\infty}\left|C_{k}\right| r^{k}<\varepsilon$,

$$
\left|H(x)+\sum_{k=0}^{n-1} p_{k} S_{k} x^{k}-P(x) T(x)\right|<\delta(x)|P(x)| \quad(r \leqslant x<\rho) .
$$

There exists a sequence $s$ that satisfies

(3.8) $s_{j}=S_{j} \quad(j=0,1, \ldots, n-1), \quad \sum_{k=n}^{\infty} p_{k} s_{k} x^{k} \equiv H(x)=\sum_{k=n}^{\infty} C_{k} x^{k}$.

Now (3.8) clearly implies (3.7) with the possible exception of the condition $\lim _{p} s=T(\rho-0)$. This condition however would follow from the condition $\left|T_{p}(s, x)-T(x)\right|<\delta(x)$ if we had assumed (with no loss of generality!) that $\delta(\rho-0)=0$.

3.9. Lemma. Let (2.1.1) be satisfied. If

$$
\int_{\alpha}^{\rho^{-}} T_{p}(s, t) d g(t)=\sum_{k=0}^{\infty} \gamma_{k} s_{k}
$$

for all $s \in c_{p}^{(0)}$ where $g$ is of bounded variation and

$$
\left|\gamma_{k}\right| \leqslant R\left|p_{k}\right| r^{k}, \quad \alpha<r<\rho(k=m, m+1, \ldots, m \in\{0,1, \ldots\}),
$$

then $g=$ const in $[r, \rho)$.

Proof. Let $T(x)$ be any function which is continuous in $[r, \rho)$ and satisfies $T(r)=T(\rho-0)=0$. From Lemma 3.8 it follows that a sequence of sequences $s^{(l)} \in c_{p}^{(0)}(l=1,2, \ldots)$ exists which satisfies

$$
\begin{gathered}
s_{0}^{(l)}=s_{1}^{(l)}=\cdots=s_{m-1}^{(l)}=0, \quad \sum_{k=0}^{\infty}\left|p_{k} s_{k}^{(l)}\right| r^{k}<1 / l, \\
\left|T_{p}\left(s^{(l)}, x\right)-T(x)\right|<1 / l \quad(r \leqslant x<\rho, l=1,2, \ldots) .
\end{gathered}
$$

It follows that the transforms $T_{p}\left(s^{(l)}, t\right)(l=1,2, \ldots)$ are uniformly bounded and we have 


$$
\begin{aligned}
\left|\int_{r}^{\rho^{-}} T(t) d g(t)\right| & =\left|\int_{r}^{\rho^{-}} \lim _{l \rightarrow \infty} T_{p}\left(s^{(l)}, t\right) d g(t)\right|=\lim _{l \rightarrow \infty}\left|\int_{r}^{\rho^{-}} T_{p}\left(s^{(l)}, t\right) d g(t)\right| \\
& =\lim _{l \rightarrow \infty}\left|\int_{\alpha}^{\rho^{-}} T_{p}\left(s^{(l)}, t\right) d g(t)\right|-\int_{\alpha}^{r}\left(\sum_{k=0}^{\infty} p_{k} s_{k}^{(l)} t^{k} / P(t) d g(t)\right) \mid \\
& =\lim _{l \rightarrow \infty}\left|\sum_{k=0}^{\infty} \gamma_{k} s_{k}^{(l)}-\sum_{k=0}^{\infty} p_{k} s_{k}^{(l)} \int_{\alpha}^{r}\left(t^{k} / P(t)\right) d g(t)\right| \\
& \leqslant \varlimsup_{l \rightarrow \infty} \sum_{k=0}^{\infty}\left|p_{k} s_{k}^{(l)}\right| M r^{k} \leqslant \varlimsup_{l \rightarrow \infty} M / l=0 .
\end{aligned}
$$

Hence $\int_{r}^{\rho^{-}} T(t) d g(t)=0$ for all continuous functions $T(t)$ which satisfy $T(r)=T(\rho-0)=0$. This implies that $g=$ const in $[r, \rho)$.

3.10. Admissible function. Let $g(t)$ be complex valued and of bounded variation in $[\alpha, \rho) . g$ will be called admissible in $c_{p}^{(0)}$ if both sides of the equality

$$
\int_{\alpha}^{\rho^{-}}\left(\sum_{k=0}^{\infty} p_{k} s_{k} t^{k} / P(t)\right) d g(t)=\sum_{k=0}^{\infty}\left[p_{k} s_{k} \int_{\alpha}^{\rho^{-}}\left(t^{k} / P(t)\right) d g(t)\right]
$$

exist and are equal for all $s \in c_{p}^{(0)}$.

Clearly $g$ is admissible if it is constant in $[r, \rho)$ for some $r<\rho$. We shall see (Lemma 3.14) that this condition is also necessary under some fairly general restrictions.

3.11. LEMMA. Let (2.1.1) be satisfied. If $g(t)$ is admissible in $c_{p}^{(0)}$ and

$$
\begin{aligned}
\left|p_{k} \int_{\alpha}^{\rho^{-}}\left(t^{k} / P(t)\right) d g(t)\right| & \leqslant R\left|p_{k}\right| r^{k}, \\
\alpha & <r<\rho(k=m, m+1, \ldots ; m \in\{0,1, \ldots\}),
\end{aligned}
$$

then $g=$ const in $\stackrel{1}{[r}, \rho)$.

Proof. Denote $\gamma_{k}=p_{k} \int_{\alpha}^{\rho^{-}}\left(t^{k} / P(t)\right) d g(t)(k=0,1,2, \ldots)$. Since (3.9) is satisfied and since $g$ is admissible we have

$$
\int_{\alpha}^{\rho^{-}} T_{p}(s, t) d g(t)=\int_{\alpha}^{\rho^{-}}\left(\sum_{k=0}^{\infty} p_{k} s_{k} t^{k} / P(t)\right) d g(t)=\sum_{k=0}^{\infty} \gamma_{k} s_{k} \quad\left(s \in c_{p}^{(0)}\right) .
$$

Hence the result follows from Lemma 3.9.

3.12. LEMMA. Let (2.1.1) be satisfied. If $\sum_{k=0}^{\infty} \gamma_{k} s_{k}=0$ for all $s \in c_{p}^{(0)}$, where $\left\{\gamma_{k}\right\}$ satisfies (3.9), then $\gamma_{k}=0(k=0,1, \ldots)$.

Proof. Had all the sequences $e^{(l)}=(0, \ldots, 0,1,0, \ldots)$ been in $c_{p}^{(0)}$ the proof would have been trivial. Nevertheless, it follows from Lemma 3.7 that analytic functions $P(z)$ exist which do not possess a limit $1 / P(\rho-0)$. For such 
a $P, e^{(l)} \notin c_{p}^{(0)}$ for some values of $l$. Hence we have to look for a different proof. Choose then (arbitrarily) numbers $n \in\{m, m+1, \ldots\}$ and $S_{0}, S_{1}, \ldots$, $S_{n-1}$. Lemma 3.8 implies the existence of sequences $s^{(l)} \in c_{p}^{(0)}(l=1,2, \ldots)$ satisfying

$$
s_{j}^{(l)}=S_{j} \quad(j=0,1, \ldots, n-1), \quad \sum_{k=n}^{\infty}\left|p_{k} s_{k}^{(l)}\right| r^{k}<1 / l \quad(l=1,2, \ldots) .
$$

We get

$$
\begin{gathered}
\sum_{k=0}^{n-1} \gamma_{k} s_{k}+\sum_{k=n}^{\infty} \gamma_{k} s_{k}^{(l)}=\sum_{k=0}^{\infty} \gamma_{k} s_{k}^{(l)}=0, \\
\left|\sum_{k=0}^{n-1} \gamma_{k} S_{k}\right| \leqslant \varlimsup_{l \rightarrow \infty} \sum_{k=n}^{\infty}\left|\gamma_{k} s_{k}^{(l)}\right| \leqslant \varlimsup_{l \rightarrow \infty} R \sum_{k=n}^{\infty}\left|p_{k} s_{k}^{(l)}\right| r^{k} \leqslant \varlimsup_{l \rightarrow \infty} R / l=0 .
\end{gathered}
$$

Hence the freedom in choosing $n$ and $S_{0}, \ldots, S_{n-1}$ implies that $\gamma_{k}=0$ for all $k$.

3.13. Proof of TheOREM 2.2.4. The set of all sequences $s$ for which $\sum \beta_{k} s_{k}$ converges coincides with the field $c_{w}$ of the Toeplitz matrix

$$
w_{n k}= \begin{cases}\beta_{k}, & k \in\{0,1, \ldots, n\}, \\ 0, & k \in\{n+1, n+2, \ldots\} .\end{cases}
$$

This field is an FK space, one of whose seminorms is $q(s)=\sup _{n}\left|\sum_{k=0}^{n} \beta_{k} s_{k}\right|$. Obviously $c_{p}^{(0)} \subseteq c_{w}$; hence its topology is stronger than that of $c_{w}$ and therefore $q(s)$ is continuous in $c_{p}^{(0)}$ and thus satisfies there

$$
\sup _{n}\left|\sum_{k=0}^{n} \beta_{k} s_{k}\right| \leqslant M\left(Q_{\infty}(s)+\sum_{k=0}^{2 m} Q(s)\right), \quad M<\infty, m \in\{0,1, \ldots\} .
$$

Using this result with an argument similar to the one used in the proof of Theorem 2.2.3 we get for the functionals $\sum_{k=0}^{n} \beta_{k} s_{k}(n=0,1, \ldots)$ representations of the form

$$
\sum_{k=0}^{n} \beta_{k} s_{k}=\sum_{k=0}^{\infty} \gamma_{n k} s_{k}+\int_{\alpha}^{\rho^{-}} T_{p}(s, t) d g_{n}(t) \quad\left(s \in c_{p}^{(0)}, n=0,1, \ldots\right)
$$

where $\gamma_{n k} \leqslant L\left|p_{k}\right| r^{k}(k=m, m+1, \ldots), \int_{\alpha}^{\rho^{-}}\left|d g_{n}(t)\right| \leqslant L$, and $L, r<\rho$ are independent of $n$. From Lemma 3.9 it follows that $g_{n}=$ const in $[r, \rho)$.

Hence

$$
\sum_{k=0}^{n} \beta_{k} s_{k}=\sum_{k=0}^{\infty}\left[\gamma_{n k}+p_{k} \int_{\alpha}^{r}\left(t^{k} / P(t)\right) d g_{n}(t)\right] s_{k} \equiv \sum_{k=0}^{\infty} \delta_{n k} s_{k} .
$$

But 
fundamental in $c_{p}^{(0)}$ and $U$ is fundamental in $c_{p}$. (b) If $e^{(l)} \notin c_{p}^{(0)}$ for at least one $l$ then $U^{\prime}$ is fundamental in $c_{p}$.

Proof. (a) We show first the fundamentality of $U$ in $c_{p}$. For this it suffices to show that every continuous linear functional $f$ in $c_{p}$ which vanishes in $U$ vanishes everywhere in $c_{p}$. From Theorem 2.2.3, $f$ has a representation of the form (2.2.1) with (2.2.2) satisfied. We substitute $s=e^{(l)}$ in (2.2.1) and get (ii) of Theorem 2.2.5. (i) of Theorem 2.2.5 follows, then from the $\left(M\left\{k_{i}\right\}\right)$ property of $P(t)$. Hence $f \equiv 0$ in $c_{p}^{(0)}$ from Theorem 2.2.5(a) (see Remark 2.2.6). Let now $s \in c_{p}$ and denote $\lim _{p} s=a$. Then $f(s)=f(s-a e)+a f(e)$ $=0+a \cdot 0$ and the fundamentality of $U$ in $c_{p}$ is proved.

Let now $s \in c_{p}^{(0)}$. There exists a sequence which tends to $s$ of sequences $s^{(n)} \in c_{p}(n=1,2, \ldots)$ which are finite linear combinations of terms of $U$. We may write $s^{(n)}=t^{(n)}+a_{n} e$ where $a_{n}=\lim _{p} s^{(n)}$. Since $\lim _{p}$ is a continuous functional we have $a_{n} \rightarrow 0$, hence $t^{(n)} \rightarrow s$. But obviously $t^{(n)} \in c_{p}^{(0)}$ are finite linear combinations of terms in $U^{\prime}$ so (a) is proved.

(b) Since $\lim _{p} e^{(l)} \equiv \lim _{x \rightarrow \rho^{-}} p_{l} x^{l} / P(x)$ is finite for all $l$ and differs from zero for some $l$ it is necessary that $\rho$ is finite and the limit $1 / P(\rho-0)$ exists and $\neq 0$. We use again representations of continuous linear functionals suggested by Theorem 2.2.3. Substituting $s=e^{(l)}(l=0,1, \ldots)$ in $(2.2 .1)$ we get

$$
\beta_{l}+p_{l} \int_{\alpha}^{\rho^{-}}\left(t^{l} / P(t)\right) d g(t)+\beta p_{l} \rho^{l} / P(\rho-0)=0 \quad(l=0,1, \ldots) .
$$

Hence by (2.2.2)

$$
p_{l}\left[o\left(\rho^{l}\right)+\beta \rho^{l} / P(\rho-0)\right]=0 \quad(l=0,1, \ldots, l \rightarrow \infty) .
$$

This implies $\beta=0$. Thus (ii) and (iii) of Theorem 2.2.5 are satisfied and we proceed as in the proof of part (a) with the aid of Theorem 2.2.5(b).

4.3. Proof OF TheOREM 2.3.2. Since $e^{(l)} \in c_{p}(l=0,1, \ldots)$,

$$
\sup _{\alpha<t<\rho}\left|t^{k} / P(t)\right|<\infty \quad(k=0,1, \ldots)
$$

and Theorem 2.3.2 follows immediately from Lemmas 4.1 and 4.2.

4.4. Proof OF TheOREM 2.3.4. The sufficiency follows from Lemma 4.2. Let us prove the necessity. Assume that

$$
\int_{\alpha}^{\rho^{-}}\left(t^{k_{i}} / P(t)\right) d g(t)=O\left(r^{k_{i}}\right) \quad\left(\alpha<r<\rho, \int_{\alpha}^{\rho^{-}}|d g(t)|<\infty, i \rightarrow \infty\right)
$$

and denote $\gamma_{k}=p_{k} \int_{\alpha}^{\rho^{-}}\left(t^{k} / P(t)\right) d g(t)(k=0,1, \ldots)$. Obviously (3.9) is satisfied. The functional

$$
f(s)=\sum_{k=0}^{\infty} \gamma_{k} s_{k}-\int_{\alpha}^{\rho^{-}} T_{p}(s, t) d g(t)
$$




$$
\left|\delta_{n k}\right| \leqslant\left|\gamma_{n k}\right|+\left|p_{k}\right| N r^{k} \int_{\alpha}^{r}\left|d g_{n}(t)\right| \leqslant L\left|p_{k} r^{k}\right|+\left|p_{k}\right| N r^{k} L \equiv R\left|p_{k}\right| r^{k} .
$$

Therefore we may deduce from Lemma 3.12 that

$$
\left|\beta_{k}\right|=\left|\delta_{n k}\right| \leqslant R\left|p_{k}\right| r^{k} \quad(k=m, m+1, \ldots, n) .
$$

Since $n$ may be arbitrarily increased (2.2.2) follows.

3.14. Lemma. Let (2.1.1) be satisfied and let $g$ be some function of bounded variation in $[\alpha, \rho) . g$ is admissible in $c_{p}^{(0)}$ if and only if $g=$ const in $[r, \rho)$ for some $r<\rho$.

Proof. Sufficiency is obvious. Let us prove necessity. We denote

$$
\beta_{k}=-p_{k} \int_{\alpha}^{\rho^{-}}\left(t^{k} / P(t)\right) d g(t) \quad(k=0,1, \ldots) .
$$

Since $g$ is admissible $\sum \beta_{k} s_{k}$ converges for all $s \in c_{p}^{(0)}$. Therefore Theorem 2.2.4 implies that (2.2.2) is satisfied. Hence it follows from Lemma 3.11 that $g=$ const in $[r, \rho)$.

3.15. Proof of Theorem 2.2.5. Sufficiency is obvious. Let us prove necessity.

(a) If $f$ is well defined in $c_{p}^{(0)}$ then, by Theorem 2.2.4, (2.2.2) is satisfied and therefore (i) follows from Lemma 3.9. Hence for all $s \in c_{p}^{(0)}$

$$
\sum_{k=0}^{\infty}\left[\beta_{k}+p_{k} \int_{\alpha}^{r}\left(t^{k} / P(t)\right) d g(t)\right] s_{k}=0
$$

which implies (ii) by Lemma 3.12 .

(b) Conditions (i), (ii) follow from (a). In order to get (iii) we substitute $s=e=(1,1, \ldots)$ in $(2.2 .1)$ and get from (i) and (ii)

$$
0=f(e)=\sum_{k=0}^{\infty} \beta_{k}+\int_{\alpha}^{\rho^{-}}\left(\sum_{k=0}^{\infty} p_{k} t^{k} / P(t)\right) d g(t)+\beta=\beta .
$$

\section{Proofs of the results in $\$ 2.3$.}

4.1. Lemma. If $0 \leqslant \alpha<\rho<\infty, \sum 1 / k_{i}=\infty$ and $\phi$ is continuous in $[\alpha, \rho)$ and satisfies (2.3.1) then $\phi$ has the $\left(M\left\{k_{i}\right\}\right)$ property in $[\alpha, \rho)$.

Proof. Define $\chi(\tau)=0$ in $[0, \alpha / \rho], \chi(\tau)=\int_{\alpha}^{\rho \tau}\left[t^{k_{0}} / \phi(t)\right] d g(t)$ in $(\alpha / \rho, 1)$, $\chi(1)=\chi(1-0)$ and use Trautner's theorem (\$3.5).

4.2. Lemma. Let $e^{(l)} \in c_{p}(l=0,1, \ldots)$ and denote $0 \leqslant k_{0}<k_{1}<\cdots$, the sequence $\left\{k_{i}\right\}=\left\{k \mid p_{k} \neq 0\right\}$, which is assumed to be infinite. Assume that $P(t)$ has the $\left(M\left\{k_{i}\right\}\right)$ property in $[\alpha, \rho)$. (a) If $e^{(l)} \in c_{p}^{(0)}(l=0,1, \ldots)$ then $U^{\prime}$ is 
is continuous in $c_{p}$ and satisfies $f\left(e^{(l)}\right)=0$. Since $\lim _{p} e^{(l)}$ is finite for all $l$ and since $\rho=\infty$ we have $\lim _{p} e^{(l)}=0(l=0,1, \ldots)$. Hence the fundamentality of $U$ in $c_{p}$ implies that $U^{\prime}$ is fundamental in $c_{p}^{(0)}$ (see proof of Lemma 4.2(a)) and therefore $f(s)=0$ for all $s \in c_{p}^{(0)}$. It follows, then, from Lemma 3.9, that $g=$ const in $[r, \rho)$.

4.5. Proof of Remark 2.3.9. Let

$$
\int_{\alpha}^{\infty-}\left(t^{k_{i}} / \phi_{2}(t)\right) d g(t)=O\left(r^{k_{i}}\right) \quad\left(i \rightarrow \infty, \alpha<r<\infty, \int_{\alpha}^{\infty-}|d g(t)|<\infty\right) .
$$

Defining

$$
h(\varepsilon t)=\int_{\alpha}^{t}\left(\phi_{1}(\varepsilon \theta) / \phi_{2}(\theta)\right) d g(\theta) \quad(\alpha \leqslant t<\infty)
$$

we get

$$
\int_{\varepsilon \alpha}^{\infty-}\left(\tau^{k_{i}} / \phi_{1}(\tau)\right) d h(\tau)=O\left[(\varepsilon r)^{k_{i}}\right] \quad(i \rightarrow \infty) .
$$

Thus the $\left(M\left\{k_{i}\right\}\right)$ property of $\phi_{1}$ implies $h(\tau)=$ const for $\tau \in[\varepsilon r,+\infty)$. Hence $g(t)=$ const for $t \in[r,+\infty)$.

4.6. Proof of Theorem 2.3.5. Since $P$ is regular, a finite number of $p_{k}$ 's might be changed without essentially affecting the method. We may assume, then, that $p_{k} \neq 0$ for all $k$. Let us denote $\hat{P}(s)=\sum_{k=0}^{\infty}\left|p_{k}\right| x^{k}$. Since $P$ is a regular method we have

$$
L \hat{P}(x) \leqslant|P(x)| \leqslant \hat{P}(x) \quad(\alpha \leqslant x<\rho)
$$

for some constant $0<L \leqslant 1$. (This follows from the well-known conditions for regularity of continuous methods. See Włodarski [17].) Hence from Remark 2.3.9 it follows that the $(M)$ properties in $[\alpha, \infty)$ of $\hat{P}$ and of $P$ are equivalent. From Birkholc's Theorem 2.3.8, this is equivalent to

$$
\int_{-\infty}^{\infty}\left[\ln \hat{P}\left(\tau^{2}\right) /\left(1+\tau^{2}\right)\right] d \tau=\infty
$$

which is equvivalent to (2.3.3).

4.7. Proof of Remark 2.3.7. Assume that either $U$ or $U^{\prime}$ is a Schauder basis and let $f$ be any continuous linear functional in $c_{p}$. Obviously

$$
\lim _{n \rightarrow \infty} f\left(s-\sum_{l=0}^{n} s_{l} e^{(l)}\right)=0 \quad\left(s \in c_{p}^{(0)}\right) .
$$

Hence $f(s)=\sum_{l=0}^{\infty} s_{l} f\left(e^{(l)}\right)\left(s \in c_{p}^{(0)}\right)$. In particular this holds for any functional of the form $f(s)=\int_{\alpha}^{\rho^{-}} T_{p}(s, t) d g(t), \int_{\alpha}^{\rho^{-}}|d g(t)|<\infty$. It means that 
every $g \in B V$ is admissible in $c_{p}^{(0)}$ which is incorrect by Lemma 3.14 .

\section{Proofs of the results in $\$ 2.4$.}

5.1. Lemma. Let $\left[E, q_{j}\right](j=0,1, \ldots)$ be an $F K$ space and define

$$
q(s)=\sup _{x \in I}\left|T_{w}(s, x)\right|=\sup _{x \in I}\left|\sum_{k=0}^{\infty} w_{x k} s_{k}\right|
$$

where $W$ is any matrix method. If the seminorm $q(s)$ is well defined and is finite for all $s \in E$ then it is continuous in $\left[E, q_{j}\right]$.

Proof. Had we shown that $\left[E, q_{j}, q\right]$ is an FK space the continuity of $q$ in $\left[E, q_{j}\right]$ would follow from the uniqueness of an FK topology in a given space. The only difficulty rests in the proof of the completeness of this space. Let, then, $\left\{s^{(l)}\right\}$ be any Cauchy sequence in the seminorms $q$ and $q_{j}(j=0,1, \ldots)$. Since $\left[E, q_{j}\right]$ is complete $\left\{s^{(l)}\right\}$ has some limit within the space, denote by $s$. All we have to show is that $q\left(s^{(l)}-s\right) \rightarrow 0(l \rightarrow \infty)$. However, $\left\{T_{w}\left(s^{(l)}, x\right)\right\}$ is a Cauchy sequence in the Banach space of all bounded functions in $I$. Hence it has a limit, $T(x)$ (the convergence is uniform). But for any $x \in I, T_{w}(\cdot, x)$ is a continuous functional in $\left[E, q_{j}\right]$. Therefore $\left.\lim _{l \rightarrow \infty} T_{w}(s), x\right)=T_{w}(s, x)$ so we have $T_{w}(s, x) \equiv T(x)$, which completes the proof.

5.2. ProOF OF THEOREM 2.4.1. Using the continuity of the seminorm $q(s)$ of Lemma 5.1 with a technique similar to that used in the proof of Theorem 2.2.4 (see §3.13) we conclude that

$$
\sum_{k=0}^{\infty} w_{x k} s_{k}=\sum_{k=0}^{\infty} d_{x k} s_{k}+\int_{\alpha}^{\rho^{-}} T_{p}(s, t) d g_{x}(t) \quad\left(s \in c_{p}^{(0)}, x \in I\right)
$$

where $\left|d_{x k}\right| \leqslant R\left|p_{k}\right| r^{k}(k=m, m+1, \ldots, m \in\{0,1, \ldots\}), \int_{\alpha}^{\rho^{-}}\left|d g_{x}(t)\right|$ $\leqslant R$. From Theorem 2.2.5(a) we get, then, that $g_{x}=$ const in $\left[r_{x}, \rho\right)$ for some $r_{x}<\rho$ and that

$$
w_{x k}=d_{x k}+p_{k} \int_{\alpha}^{r_{x}}\left(t^{k} / P(t)\right) d g_{x}(t)
$$

which completes the proof.

5.3. LEMMA. Let (2.1.1) be satisfied and assume that $\lim _{w} e^{(l)}=0(l=0$, $1, \ldots)$ and $\lim _{w} e \neq 0$. If $c_{p} \subseteq m_{w}$ then $\lim _{x \rightarrow x_{0}} w_{x}=\rho(x \in I)$ where

$$
w_{x}=\varlimsup_{k \rightarrow \infty} \sqrt[k]{\left|w_{x k} / p_{k}\right|} \quad(x \in I)
$$

and only values of $k$ with $p_{k} \neq 0$ are considered.

Proof. If $c_{p} \subseteq m_{w}$ then $\sum_{k} w_{x k} s_{k}$ converges for all $s \in c_{p}$ and $x \in I$. 
Therefore by Theorem 2.2.4, $w_{x}<\rho(x \in I)$. Hence $\overline{\lim }_{x \rightarrow x_{0}} w_{x} \leqslant \rho(x \in I)$. Assume that $\underline{\lim }_{x \rightarrow x_{0}} w_{x}<\rho(x \in I)$. This implies the existence of a subset $J$ of $I$ which has $x_{0}$ as accumulation point and satisfies $\left|w_{x k}\right| \leqslant L_{x}\left|p_{k}\right| a^{k}, a<\rho$ $\left(x \in J, p_{k} \neq 0\right)$. By Theorem 2.4.1 we have, then, for $k \geqslant m$ :

$$
\left|c_{x k}\right|=\left|p_{k} \int_{\alpha}^{r_{x}}\left(t^{k} / P(t)\right) d g_{x}(t)\right| \leqslant L_{x}\left|p_{k}\right| a^{k}+R\left|p_{k}\right| r^{k}, \quad a<\rho, r<\rho .
$$

Hence by Lemma 3.11 we see that actually $\left|c_{x k}\right| \leqslant M\left|p_{k}\right| b^{k}, b=\max \{a, r\}$ $<\rho(x \in J, k=0,1, \ldots)$ where $M$ is independent of $x$. So we may write

$$
\begin{aligned}
\left|w_{x k}\right| \leqslant\left|c_{x k}\right|+\left|d_{x k}\right| \leqslant(M+R)\left|p_{k}\right| b^{k} & =N\left|p_{k}\right| b^{k} \\
(x & \in J, k=m, m+1, \ldots)
\end{aligned}
$$

and $N, b$ are independent of both $k$ and $x$. Thus for all $s \in c_{p}$-considering values of $x$ in $J$ only-we get

$$
\lim _{w} s=\lim _{x \rightarrow x_{0}} \sum_{k=0}^{\infty} w_{x k} s_{k}=\sum_{k=0}^{\infty} \lim _{x \rightarrow x_{0}} w_{x k} s_{k}=\sum_{k=0}^{\infty}\left(\lim _{w} e^{(k)}\right) s_{k}=0
$$

where the limit-summation exchange is permitted because $\sum w_{x k} s_{k}$ is majorized by the convergent series $\sum N\left|p_{k} s_{k}\right| b^{k}$. This contradicts the fact that $\lim _{w} e \neq 0$ and completes the proof.

5.4. Proof of TheOREm 2.4.4. Assume that such a matrix method does exist. From Lemma 5.3 it follows, then, that $\lim _{x \rightarrow x_{0}} w_{x}=\rho(x \in I)$. However, this is impossible since $w_{x}=0$ for all $x$ because $W$ is raw-finite.

5.5. Proof of Corollary 2.4.5. Assume that such a series-to-sequence Toeplitz method, for example $V$, exists. The $V$ transform of a sequence $s=\left\{s_{k}\right\}$ is $\sum_{l=0}^{\infty} v_{n l} a_{l}(n=1,2, \ldots)$ where $a_{l}=s_{l}-s_{l-1}$. Since $V$ is rawfinite we may write

$$
\sum_{l=0}^{\infty} v_{n l} a_{l}=\sum_{k=0}^{\infty}\left(v_{n k}-v_{n, k+1}\right) s_{k}
$$

and we see that the $V$-transform of $s$ is actually identical with the $W$-transform of $s$ where $W$ is the raw-finite and regular sequence-to-sequence Toeplitz method with the matrix $w_{n k}=v_{n k}-v_{n, k+1}$. Obviously $c_{p}^{(0)} \subseteq m_{w}$ which is in contradiction with Theorem 2.4.4.

5.6. LEMMA. Let $E$ be an FK space and let $\left\{f_{n}\right\}$ be a sequence of continuous linear functionals on $E$. If $\lim _{n \rightarrow \infty} f_{n}(s)$ exists for all terms s of some fundamental set in $E$ and if the sequence $\left\{f_{n}(s)\right\}$ is bounded for all $s \in E$ then the limit $f(s)=\lim _{n \rightarrow \infty} f_{n}(s)$ exists for all $s \in E$ and is a continuous linear functional in E. 
Proof. For proof of a more general theorem see Dunford and Schwartz [7, II, 1.18]. See also Köthe [9].

5.7. Proof of TheOREM 2.4.6. The necessity follows immediately from the fact that $U \subseteq c_{p} \subseteq c_{w}$. The sufficiency follows from Lemma 5.6 by consideration of the continuous linear functionals

$$
f_{x}(s)=\sum_{k=0}^{\infty} w_{x k} s_{k} \quad(x \in I) .
$$

5.8. Proof of Remark 2.4.7. We use a theorem by L. Włodarski [17, Theorem VI(b)] which states the following: Let $\Lambda$ be a continuous method of limitation. For every additive and continuous functional $f(s)$ on the field $c_{\lambda}$ of $\Lambda$ there exists a Toeplitz method $Z$ such that $c_{\lambda} \subseteq c_{z}$ and $\lim _{z} s=f(s)$ for all $s \in c_{\lambda}$.

Let us prove Włodarski's theorem for the limited case where $\Lambda=P$ is a power method and the functional $f$ is linear:

From Theorem 2.2.3 $f$ has a representation of the form (2.2.1). We may write, then,

$$
f(s)=\sum_{k=0}^{\infty} \beta_{k} s_{k}+\lim _{j \rightarrow \infty} \int_{\alpha}^{\lambda_{j}} T_{p}(s, t) d g(t)+\beta \lim _{j \rightarrow \infty} T_{p}\left(s, \lambda_{j}\right)
$$

where $\alpha<\lambda_{1}<\lambda_{2}<\cdots<\lambda_{j} \rightarrow \rho$. Hence

$$
\begin{aligned}
f(s)= & \sum_{k=0}^{\infty} \beta_{k} s_{k}+\lim _{j \rightarrow \infty} \sum_{k=0}^{\infty} p_{k} s_{k} \int_{\alpha}^{\lambda_{j}}\left(t^{k} / P(t)\right) d g(t) \\
& +\lim _{j \rightarrow \infty} \sum_{k=0}^{\infty} \beta p_{k} s_{k} \lambda_{j}^{k} / P\left(\lambda_{j}\right)
\end{aligned}
$$

and we see that acutally $f(s)=\lim _{z} s$ where

$$
\begin{aligned}
z_{j k}=\beta_{k}+p_{k} \int_{\alpha}^{\lambda_{j}}\left(t^{k} / P(t)\right) d g(t) & +\beta p_{k} \lambda_{j}^{k} / P\left(\lambda_{j}\right) \\
& (j=1,2, \ldots, k=0,1, \ldots) .
\end{aligned}
$$

Włodarski's theorem is thus proved.

Now, if $U$ is not fundamental in $c_{p}$ there exists a continuous linear functional $f$, which vanished over $U$ but not completely over $c_{p}$. By Whodarski's theorem there exists a Toeplitz matrix $Z$ such that $\lim _{z} s=f(s)$ for all $s \in c_{p}$. We split the index set $I$ into two disjoint subsets- $I=J \cup K$-in such a way that $J$ is composed of one convergent sequence $x_{j} \rightarrow x_{0}$ and $K$ has $x_{0}$ as an accumulation point. Then we define a matrix

$$
w_{x k}= \begin{cases}z_{j k}, & x=x_{j} \in J, \\ 0, & x \in K\end{cases}
$$


Obviously $c_{p} \subseteq m_{w}$ and therefore conditions (i), (ii) of Theorem 2.4.1 are satisfied. Moreover, conditions (iii), (iv) of Theorem 2.4.6 are satisfied also because, for all $s \in U, \lim _{z} s=f(s)=0$; hence $\lim _{w} s=0$. However $c_{p}$ $\nsubseteq c_{w}$ because $s \notin c_{w}$ for all $s \in c_{p}$ such that $f(s) \neq 0$.

5.9. LeMma. Let $\left\{\left(g_{x}, d_{x}\right) \mid x \in I\right\}$ be a set of systems whose components $g_{x}=g_{x}(t), d_{x}=\left\{d_{x k}\right\}$ satisfy all the conditions mentioned in (i) and (ii) of Theorem 2.4.1 and assume further that $g_{x}=0$ in $\left[r_{x}, \rho\right]$. For every sequence $x \in I(n=1,2, \ldots)$ such that $x_{n} \rightarrow x_{0}$ the set $\left\{\left(g_{x_{n}}, d_{x_{n}}\right) \mid n=1,2, \ldots\right\}$ has at least one accumulation system at $x_{0}$.

Proof. This is an immediate result of the principle of choice. (See Widder [15, Chapter I, Theorems 16.1 and 16.3]. It should be emphasized that Theorem 16.3 is also true for the case of an infinite interval, provided this interval is closed at both ends.)

5.10. LemMa. Let $\left\{\left(g_{x}, d_{x}\right) \mid x \in I\right\}$ be the set of systems of Lemma 5.9. If $x_{n} \in I \quad(n=1,2, \ldots)$ is such that $x_{n} \rightarrow x_{0}, g_{x_{n}}(t) \rightarrow g(t), d_{x_{n}, k} \rightarrow d_{k}(n$ $\rightarrow \infty, \alpha \leqslant t<\rho, k=0,1, \ldots)$ then

$$
\lim _{n \rightarrow \infty} T_{w}\left(s, x_{n}\right)=\sum_{k=0}^{\infty} d_{k} s_{k}+\int_{\alpha}^{\rho^{-}} T_{p}(s, t) d g(t)-g(\rho-0) \lim _{p} s
$$

$$
\begin{aligned}
& \int_{\alpha}^{\rho^{-}}|d g(t)|+|g(\rho-0)| \leqslant R, \\
&\left|d_{0}\right|,\left|d_{1}\right|, \ldots \leqslant R,\left|d_{k}\right|<R\left|p_{k}\right| r^{k} \quad(k=m, m+1, \ldots) .
\end{aligned}
$$

Proof.

$$
\begin{aligned}
\lim _{n \rightarrow \infty} T_{w}\left(s, x_{n}\right) & =\lim _{n \rightarrow \infty} \sum_{k=0}^{\infty}\left(c_{x_{n}, k}+d_{x_{n}, k}\right) s_{k} \\
& =\lim _{n \rightarrow \infty} \sum_{k=0}^{\infty} p_{k} s_{k} \int_{\alpha}^{\rho}\left(t^{k} / P(t)\right) d g_{x_{n}}(t)+\lim _{n \rightarrow \infty} \sum_{k=0}^{\infty} d_{x_{n}, k} s_{k} \\
& \equiv l_{1}+l_{2} .
\end{aligned}
$$

Interchanging ' $S$ ' and ' $\Sigma$ ' in $l_{1}$, which is permissible because $g_{x_{n}}=$ const in some neighborhood of $\rho$, we get

$$
l_{1}=\lim _{n \rightarrow \infty} \int_{\alpha}^{\rho} T_{p}(s, t) d g_{x_{n}}(t) .
$$

Since $T_{p}(s, t)$ is continuous in $[\alpha, \rho]$ (put $\left.T_{p}(s, \rho)=\lim _{p} s\right)$ and since $g_{x_{n}}(n$ $=1,2, \ldots$ ) have uniformly bounded variations ' $f$ ' and 'lim' may also be 
interchanged and therefore

$$
l_{1}=\int_{\alpha}^{\rho} T_{p}(s, t) d g(t)=\int_{\alpha}^{\rho^{-}} T_{p}(s, t) d g(t)-g(\rho-0) \lim _{p} s .
$$

(See Widder [15, Chapter I, Theorem 16.4]. Notice that this theorem is also true for an infinite interval provided that it is closed.)

In $l_{2}$ the limit may be introduced into the ' $\Sigma$ ' because this sum is majorized by $\sum_{k} R\left|p_{k} s_{k}\right| r^{k}$ which is convergent. Thus (5.1) is proved. (5.2) is deduced by Corollary 16.4 in Widder [15, Chapter I].

5.11. Proof of Theorem 2.4.12. Necessity. Assume that $c_{p} \subseteq c_{w}$. By Lemma 5.9 there exists at least one accumulation system $\left(g^{\prime}, d^{\prime}\right)$. Define $\tilde{g}(t)=g^{\prime}(t) ; \tilde{d}_{k}=d_{k}^{\prime}+p_{k} \int_{\alpha}^{r+}\left(t^{k} / P(t)\right) d g^{\prime}(t)$. Let now $(g, d)$ be any other accumulation system. From Lemma 5.10, (2.4.1) and (2.4.2) follow. Analogous results for $\left(g^{\prime}, d^{\prime}\right)$ are also true. Hence

$$
\begin{aligned}
\sum_{k=0}^{\infty} d_{k} s_{k}+\int_{\alpha}^{\rho^{-}} T_{p}(s, t) d g(t)-g(\rho-0) \lim _{p} s & \\
= & \sum_{k=0}^{\infty} d_{k}^{\prime} s_{k}+\int_{\alpha}^{\rho^{-}} T_{p}(s, t) d g^{\prime}(t)-g^{\prime}(\rho-0) \lim _{p} s \quad\left(s \in c_{p}\right) .
\end{aligned}
$$

From Theorem 2.2.5 it follows, then, that $g-g^{\prime}=$ const in some neighborhood of $\rho$,

$$
\begin{array}{rl}
\left|p_{k} \int_{\alpha}^{\rho^{-}}\left(t^{k} / P(t)\right) d\left(g(t)-g^{\prime}(t)\right)\right|=\left|d_{k}-d_{k}^{\prime}\right| \leqslant 2 & 2 R\left|p_{k}\right| r^{k} \\
& (k=m, m+1, \ldots)
\end{array}
$$

and $g(\rho-0)-g^{\prime}(\rho-0)=0$. Hence (i) follows from Lemma 3.11. From (5.3) we get then

$$
\begin{aligned}
\sum_{k=0}^{\infty} d_{k} s_{k}+ & \sum_{k=0}^{\infty} p_{k} s_{k} \int_{\alpha}^{r+}\left(t^{k} / P(t)\right) d g(t) \\
& =\sum_{k=0}^{\infty} d_{k}^{\prime} s_{k}+\sum_{k=0}^{\infty} p_{k} s_{k} \int_{\alpha}^{r+}\left(t^{k} / P(t)\right) d g^{\prime}(t)=\sum_{k=0}^{\infty} \tilde{d}_{k} s_{k}
\end{aligned}
$$

which implies (ii) by Lemma 3.12.

Sufficiency. From Lemma 5.9 it follows that $\left\{g_{x} \mid x \in I\right\}$ has at least one accumulation function at $x_{0}$. We may assume with no loss of generality that $\tilde{g}$ is that accumulation function and hence has finite variation. In order to finish the proof it suffices to show that every sequence $x_{n} \in I(n=1,2, \ldots)$ such that $x_{n} \rightarrow x_{0}$ includes a subsequence $x_{n_{i}}(i=1,2, \ldots)$ which satisfies for all $s \in c_{p}$ 


$$
T_{w}\left(s, x_{n_{i}}\right) \rightarrow \sum_{k=0}^{\infty} \tilde{d}_{k} s_{k}+\int_{r+}^{\rho^{-}} T_{p}(s, t) d \tilde{g}(t)-\tilde{g}(\rho-0) \lim _{p} s .
$$

But this follows naturally from Lemmas 5.9 and 5.10.

5.12. Proof of Remark 2.4.13. Let the rational numbers in $(\alpha, \rho)$ be ordered in a sequence of reduced fractions: $j_{n} / q_{n}(n=1,2, \ldots)$. We define

$$
g_{n}(t)= \begin{cases}1, & t \in\left[\left(j_{n}-1\right) / q_{n}, j_{n} / q_{n}\right), \quad(n=1,2, \ldots) . \\ 0, & \text { elsewhere, }\end{cases}
$$

It is not difficult to see that an accumulation function of $\left\{g_{n} \mid n=1,2, \ldots\right\}$ may differ from zero at most at one point in $(\alpha, \rho)$. Thus condition (i) of Thoerem 2.4.12 is satisfied with $\tilde{g}=0$. However every function of the type

$$
g(t)=\left\{\begin{array}{ll}
1, & t=\sigma, \\
0, & t \neq \sigma,
\end{array} \quad \alpha<\sigma<\rho,\right.
$$

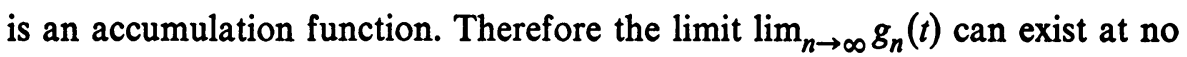
point $t \in(\alpha, \rho)$.

5.13. Proof of TheOREM 2.4.15. The sufficiency follows immediately from Remark 2.4.2. Let us prove the necessity:

If $c_{p} \subseteq m_{w}$ then $W=C^{\prime}+D^{\prime}$ where the matrices $C^{\prime}=\left(c_{x k}^{\prime}\right), D^{\prime}=\left(d_{x k}^{\prime}\right)$ satisfy conditions analogous to (i), (ii) of Theorem 2.4.1 (with $r_{x}^{\prime}, g_{x}^{\prime}(t), R^{\prime}, r^{\prime}$, $m^{\prime}$ instead of $\left.r_{x}, g_{x}(t), \ldots\right)$. With no loss of generality we may assume further that $g_{x}^{\prime} \equiv 0$ in $\left[r_{x}^{\prime}, \rho\right](x \in I)$. Let us define:

$R$-sufficiently large, $r_{x}=r_{x}^{\prime}(x \in I), r=r^{\prime}, m=m^{\prime}$

$$
\begin{aligned}
g_{x}(t) & =\left\{\begin{array}{ll}
0, & \alpha \leqslant t<r \\
g_{x}^{\prime}(t), & r \leqslant t \leqslant \rho
\end{array} \quad(x \in I),\right. \\
c_{x k} & =p_{k} \int_{\alpha}^{r_{x}}\left(t^{k} / P(t)\right) d g_{x}(t) \quad(x \in I, k=0,1, \ldots), \\
d_{x k} & =p_{k} \int_{\alpha}^{r_{x}}\left(t^{k} / P(t)\right) d g_{x}^{\prime}(t)-p_{k} r^{k} g_{x}^{\prime}(r) / P(r)+d_{x k}^{\prime} \\
& (x \in I, k=0,1, \ldots) .
\end{aligned}
$$

A straightforward calculation shows that these values satisfy: $c_{x k}+d_{x k}$ $=c_{x k}^{\prime}+d_{x k}^{\prime}=w_{x k}(x \in I, k=0,1, \ldots)$ and the conditions (i), (ii) of Theorem 2.4.1. Thus we have only to show that $c_{p} \subseteq c_{c} \cap c_{d}$. Now, since $c_{p} \subseteq c_{w}$, Theorem 2.4.12 implies that every two accumulation functions of $\left\{g_{x} \mid x \in I\right\}$ are equal almost everywhere in $(r, \rho)$. Since both must vanish in $[\alpha, r)$, an equality at $\alpha$ and almost everywhere in $(\alpha, \rho)$ follows. Hence Corollary 
2.4.14(a) implies that $c_{p} \subseteq c_{c}$, and since $c_{p} \subseteq c_{w}, W=C+D$ we get $c_{p}$ $\subseteq c_{d}$, which completes the proof.

\section{REFERENCES}

1. A. Amir (Jakimovski), Some relations between the methods of summability of Abel, Borel, Cesàro, Hölder and Hausdorff, J. Analyse Math. 3 (1954), 346-381. MR 16, 28.

2. A. Birkholc, On generalized power methods of limitation, Studia Math. 27 (1966), 213-245. MR 34 \# 4748.

3. - Remarks on a moment problem and a problem of perfection of power methods of limitation, Colloq. Math. 24 (1971/72), 89-94. MR 46 \#5958.

4. D. Borwein, On a scale of Abel-type summability methods, Proc. Cambridge Philos. Soc. 53 (1957), 318-322. MR 19, 134.

5. $\ldots$ A logarithmic method of summability, J. London Math. Soc. 33 (1958), 212-220. MR 21 \#243.

6. - On methods of summability based on integral functions. II, Proc. Cambridge Philos. Soc. 56 (1960), 125-131. MR 22 \#6957.

7. N. Dunford and J. T. Schwartz, Linear operators. Part I, Interscience, New York, 1958. MR $22 \# 8302$.

8. K. Knopp, Theory and application of infinite series, Blackie, London, 1961.

9. G. Köthe, Topologische lineare Räume. I, Springer-Verlag, Berlin, 1960. MR 24 \# A411.

10. M. Lazić, Sur les procédés fonctionnels (de limitation), Mat. Vesnik 6(21) (1969), 425-436. MR 41 \# 4052.

11. J. G. Mikusinski, Remarks on the moment problem and a theorem of Picone, Colloq. Math. 2 (1951), 138-141. MR 13, 214.

12. C. Ryll-Nardzewski, The Borel method of limitation is perfect, Bull. Acad. Polon. Sci. Sér. Sci. Math. Astronom. Phys. 10 (1962), 649-650. MR 28 \#385.

13. P. C. Tonne, Matrix transformations on the power-series convergent on the unit disc, J. London Math. Soc. (2) 4 (1972), 667-670. MR 45 \# 4007.

14. R. Trautner, Density properties of Hausdorff moment sequences, Tôhoku Math. J. (2) 24 (1972), 347-352. MR 49 \#9558.

15. D. V. Widder, The Laplace transform, Oxford, 1946.

16. A. Wilansky, Functional analysis, Blaisdell, New York, 1964. MR 30 \#425.

17. L. Włodarksi, Sur les méthodes continues de limitation. I, Studia Math. 14 (1954), 161-187 (1955). MR 16, 814.

18. K. Zeller, Allgemeine Eigenschaften von Limitierungsverfahren, Math. Z. 53 (1951), 463-487. MR 12, 604.

19. — FK-Rüume in der Funktionentheorie. I, Math. Z. 58 (1953), 288-305. MR 14, 1092.

20. __, FK-Räume in der Funktionentheorie. II, Math. Z. 58 (1953), 414-435. MR 15, 134, p. 1139.

21. - Sur la méthode de sommation d'Abel, C. R. Acad. Sci. Paris 236 (1953), 568-569. MR 14, 744.

22. - Vergleich des Abelverfahrens mit gewöhnlichen Matrixverfahren, Math. Ann. 131 (1956), 253-257. MR 18, 301.

23. A. Ziv, Rate of growth and convergence factors for power methods of limitation, Proc. Cambridge Philos. Soc. 76 (1974), 241-246.

IBM Israel Scientific Center, Computer Science Building, Techion City, Haifa, Israel 